\title{
Reduced-Complexity Noncoherently Detected Differential Space-Time Shift Keying
}

\author{
Chao Xu, Student Member, IEEE, Shinya Sugiura, Member, IEEE, Soon Xin Ng, Senior Member, IEEE, and \\ Lajos Hanzo, Fellow, IEEE
}

\begin{abstract}
Motivated by the recent development of spatial modulation (SM) and differential space-time shift keying (DSTSK), we propose a reduced-complexity conventional differential detector (CDD) as well as its reduced-complexity multiple-symbol differential sphere detector (MSDSD) counterpart for DSTSK. Both schemes operate on a symbol-by-symbol basis in order to reduce the complexity of the classic block-by-block-based CDD and MSDSD, whilst still attaining the optimum performance of the full-search-based ML detector.
\end{abstract}

Index Terms-Differential space-time shift keying, multiple-symbol differential sphere detection, reduced complexity detection.

\section{INTRODUCTION}

$\mathbf{S}$ PATIAL modulation (SM) was introduced in [1], where only one out of the $M$ transmit antennas (TAs) was activated, hence a low-complexity single-antenna-based detector was employed. A new differential space-time modulation (DSTM) scheme dispensing with channel estimation and termed as differential space-time shift keying (DSTSK) was proposed in [2] based on the amalgamation of SM and differential linear dispersion codes (DLDC) [3]. More specifically, one out of $Q$ dispersion matrices is activated to disperse a single symbol to $M$ TAs as well as to $T$ time-slots, so that the diversity gain can be achieved. As a benefit, high-rate transmission can be attained by increasing the number of dispersion matrices, instead of increasing the number of TAs.

It was demonstrated in [4] that the symbol-by-symbol decoded DSTM design considerably reduced the decoding complexity. In this contribution, we will demonstrate that the detection of DSTSK can be carried out on a symbol-by-symbol basis using a reduced complexity SM (RC-SM) decoder. However, it was demonstrated in [5] that unless we appropriately normalize the transmit power, the optimal ML performance of SM detection cannot be achieved, because the erroneous decisions concerning the specific index of the activated antenna would

Manuscript received October 26, 2010; revised December 02, 2010; accepted December 09, 2010. Date of publication December 17, 2010; date of current version January 17, 2011. This work was supported by the European Union's Seventh Framework Programme ([FP7/2007-2013]) under Grant 214625, the RC-UK of the India-UK Advanced Technology Centre and by the China-UK 4th generation wireless systems project. The associate editor coordinating the review of this manuscript and approving it for publication was Dr. Yong Liang Guan.

C. Xu, S. X. Ng, and L. Hanzo are with the School of Electronics and Computer Science, University of Southampton, Southampton SO17 1BJ, U.K. (e-mail: cx1g08@ecs.soton.ac.uk;sxn@ecs.soton.ac.uk; lh@ecs.soton.ac.uk).

S. Sugiura is with the School of Electronics and Computer Science, University of Southampton, Southampton SO17 1BJ, U.K. He is now with the Toyota Central R\&D Labs, Inc., Aichi 480-1192, Japan (e-mail: sugiura@ ieee.org).

Digital Object Identifier 10.1109/LSP.2010.2100095 mislead the single-antenna-based detector. Against this background, the novel contributions of this letter are as follows.

1) In Section II, we further develop the DSTSK of [2] for avoiding the nonlinear Cayley transform.

2) The RC-SM detector of [1] is carefully improved in Section III for facilitating the conventional differential detection (CDD), which operates on a symbol-by-symbol basis, while attaining the optimum ML performance.

3) In Section IV, we mitigate the potential performance degradation of DSTSK in rapidly fading channels with the aid of multiple-symbol differential sphere detection (MSDSD), which operates the block-based MSDSD of [6] on a symbol-by-symbol basis.

The following notations are used throughout the paper. A DSTSK scheme employing $L$-level PSK signalling is denoted by the nomenclature of DSTSK(MNTQ)-LPSK, where $N$ indicates the number of receive antennas. Furthermore, $N_{w}$ refers to the detection window width of the MSDSD.

\section{DifFEREntial Space-Time ShIFT Keying}

The differential encoding process of DSTM is expressed as

$$
\mathbf{S}_{n}=\mathbf{X}_{n-1} \mathbf{S}_{n-1}
$$

where the transmission matrix $\mathbf{S}_{n}$ has a size of $(T \times M)$ elements, and the $(T \times T)$-element matrix $\mathbf{X}_{n}$ in (1) stores the source information, which is encoded by

$$
\mathbf{X}_{n}=\widetilde{\mathbf{A}}_{q} x_{n}
$$

where $\log _{2} Q$ bits are assigned to decide which one of the $Q$ dispersion matrices of $\left\{\widetilde{\mathbf{A}}_{q}\right\}_{q=1}^{Q}$ is activated, while $\log _{2} L$ bits are mapped to the $L$-PSK symbol $x_{n}$. Hence a DSTSK information matrix $\mathbf{X}_{n}$ conveys $\log _{2}(Q L)$ bits in total.

The DSTM differential encoding process of (1) requires $\mathbf{X}_{n}$ to be unitary [7]. The DSTSK designed in [2] proposed to generate the dispersion matrices $\left\{\widetilde{\mathbf{A}}_{q}\right\}_{q=1}^{Q}$ in (2) as Hermitian matrices, while the modulated symbols $x_{n}$ was real-valued BPSK/L-PAM, so that the dispersed Hermitian matrix $\mathbf{X}_{n}$ may be converted to a unitary matrix by the Cayley transform. However, it was recognized in [2], [3] that the nonlinearity of the Cayley transform results in a performance degradation . Therefore in this letter, the dispersion matrices $\left\{\widetilde{\mathbf{A}}_{q}\right\}_{q=1}^{Q}$ are designed to be unitary matrices directly, and the Cayley transform is deleted. Observe in (2) that the modulated symbol $x_{n}$ can be drawn from the complex-valued $L$-PSK constellations ${ }^{2}$, while the dispersed matrix $\mathbf{X}_{n}$ in (2) remains unitary.

${ }^{1}$ If QAM schemes are employed, then similar to the Differential STBCs (DSTBCs) employing QAM in [8], transmit power normalization is required in (1). Furthermore, this power normalization factor has to be known at the receiver, which in practice requires the periodic transmission of side-information. 


\section{CONVEnTIONAL DifFERENTIAL DETECTION}

\section{A. Block-Based CDD}

The resultant received signal may then be modelled as

$$
\mathbf{Y}_{n}=\mathbf{S}_{n} \mathbf{H}_{n}+\mathbf{V}_{n}
$$

where the $(T \times N)$-element matrix $\mathbf{Y}_{n}$ models the signal received. The Additive White Gaussian Noise (AWGN) term $\mathbf{V}_{n}$ has a size of $(T \times N)$ with a zero mean and a variance of $N_{0}$. The Rayleigh fading channel matrix $\mathbf{H}_{n}$ has $(M \times N)$ elements, which are generated according to Clarke's fading model. Assuming that every two consecutive fading channel matrices are nearly invariant, i.e., we have $\mathbf{H}_{n+1} \approx \mathbf{H}_{n}$, then the maximum likelihood (ML) CDD is given by [7]

$$
\mathbf{X}_{n}=\arg \min _{\mathbf{X}_{n}}\left\|\mathbf{Y}_{n+1}-\mathbf{X}_{n} \mathbf{Y}_{n}\right\|^{2} \text {. }
$$

The classic CDD of (4) is block-based, which means that all $Q L$ legitimate codewords have to be checked. If we quantify the decoding complexity in terms of the total number of complex additions, multiplications and absolute value calculations, then the complexity of the block-based CDD may be represented by $[(2 T+1) N T] \cdot(L Q)$.

\section{B. Modified CDD}

The motivation of developing a low-complexity detector is to reduce the complexity of the block-based CDD of (4) from the order of $L Q$ to the order of $(L+Q)$ by decoding $q$ and $x_{n}$ separately. The first step of simplifying the block-based CDD is to express the decision metric of (4), in a vectorial form, which may be formulated as [2]

$$
\left\{q, x_{n}\right\}=\arg \min _{\left\{q, x_{n}\right\}}\left\|\overline{\mathbf{Y}}_{n}-\overline{\mathbf{H}}_{n} \chi \overline{\mathbf{K}}_{n}\right\|^{2}
$$

where the notations are given by

$$
\begin{aligned}
\overline{\mathbf{Y}}_{n} & =\operatorname{rvec}\left(\mathbf{Y}_{n+1}\right), \\
\overline{\mathbf{H}}_{n} & =\mathbf{I}_{\mathbf{T}} \otimes \mathbf{Y}_{n}^{T}, \\
\chi & =\left[\operatorname{rvec}\left(\widetilde{\mathbf{A}}_{1}\right) \cdots \operatorname{rvec}\left(\widetilde{\mathbf{A}}_{Q}\right)\right], \\
\overline{\mathbf{K}}_{n} & =[\underbrace{0 \cdots 0}_{q-1}, x_{n}, \underbrace{0 \cdots 0}_{Q-q}]
\end{aligned}
$$

where the operation $\operatorname{rvec}(\cdot)$ takes the successive rows of the matrix considered, in order to form a vector, while $\otimes$ denotes the Kronecker product. In the absence of the Cayley transform in (2), the linearization operation seen in (5) does not impose any performance penalty [2].

It may be readily shown that the Euclidean norm calculation of (5) leads to the following decision variable:

$$
\mathbf{Z}_{n}=\left(\overline{\mathbf{H}}_{n} \chi\right)^{H} \overline{\mathbf{Y}}_{n}
$$

where $\mathbf{Z}_{n}$ is a Q-component vector, which is equivalent to a SM scheme [1] having $Q$ TAs. Therefore, the RC-SM detector proposed in [1] can be employed for detecting DSTSK.

The simplified CDD first makes a decision concerning the index $q$ by testing which particular element of the vector has the highest power. This may be expressed as

$$
q=\arg \max \left|\mathbf{Z}_{n}\right|
$$

Then a $L$-PSK demodulator is invoked to demodulate the $q$-th symbol in $\mathbf{Z}_{n}$, which may be written as

$$
x_{n}=\operatorname{round}\left(\angle Z_{n}^{q}\right) .
$$

However, it was recognize in [5] that the simplified detector of (8) and (9) cannot approach the ML decoding capability of (4). For example, given $\mathbf{Z}_{n}=[2.2+3.0 j,-3.4+1.1 j]^{T}$ and assuming that QPSK was employed, the simplified CDD of (8) and (9) would make a decision of $\left\{q, x_{n}\right\}=\{1, j\}$, but the block-based ML CDD of (5) gives $\left\{q, x_{n}\right\}=\{2,-1\}$. The problem of the simplified CDD was that the erroneous decision concerning the index $q$ made by (8) misled the the $L$-PSK demodulator of (9) to detect the wrong symbol.

Since the QPSK constellation set is $\{ \pm 1, \pm j\}$, the estimation of index $q$ would become more reliable, if the decoder could aim for finding the specific symbol, which has the highest absolute real or imaginary value. Revisiting the previous example, the index $q=2$ would be selected with a absolute real value of 3.4. Then (9) is invoked to demodulate $Z_{n}^{2}$ yielding -1 , which is the same as the ML CDD decoding result. Therefore, the ML CDD's decoding capability can be retained by using the appropriate objective function for (8).

In summary, we propose to modify (8), explicitly depending on $L$, as follows:

$$
\begin{aligned}
& L=1, \quad q=\arg \max \operatorname{Re}\left(\mathbf{Z}_{n}\right), \\
& L=2, \quad q=\arg \max \left|\operatorname{Re}\left(\mathbf{Z}_{n}\right)\right|, \\
& L=4, \quad q=\arg \max \left\{\left|\operatorname{Re}\left(\mathbf{Z}_{n}\right)\right|,\left|\operatorname{Im}\left(\mathbf{Z}_{n}\right)\right|\right\}, \\
& L=8, \quad q=\arg \max \{\left|\operatorname{Re}\left(\mathbf{Z}_{n}\right)\right|,\left|\operatorname{Im}\left(\mathbf{Z}_{n}\right)\right|, \\
&\left.\frac{\left|\operatorname{Re}\left(\mathbf{Z}_{n}\right)\right|+\left|\operatorname{Im}\left(\mathbf{Z}_{n}\right)\right|}{\sqrt{2}}\right\} .
\end{aligned}
$$

The decoding complexity of our modified CDD is constituted by two distinct contributions. Quantitatively, the first part is represented by $\left(2 T^{2}-1\right) N T Q+(2 N T-1) Q$ imposed by calculating $\mathbf{Z}_{n}$ in (7), while the second part arises from the simplified decoding operation of (9) and (10), which is given by $Q,(Q+L)$, $(2 Q+L)$ and $(3 Q+L)$ for employing 1-PSK ${ }^{2}$, BPSK, QPSK and $8 \mathrm{PSK}$, respectively.

\section{Simulation Results}

The BER performance of CDD aided DSTSK is characterized in Fig. 1(a). Observe that employing the simplified CDD adopted from [1] of (8) and (9) results in a degraded performance, regardless of which particular $L$-PSK scheme is employed. By contrast, the proposed CDD of (9) and (10) exhibits optimum ML detection capability.

The complexity comparison between the ML CDD of (4) and the proposed CDD of (9) and (10) is portrayed in Fig. 1(b). Observe in Fig. 1(b) that the overall complexity is only modestly reduced, when employing 1-PSK or BPSK. However, in the case of QPSK and 8PSK, the attainable complexity reduction becomes significant. Observe furthermore in Fig. 1(b) that

\footnotetext{
${ }^{2}$ The employment of a 1-PSK modulation scheme implies that only the antenna activation index conveys the source information, which refers to the Space-Shift Keying Scheme of [9].
} 

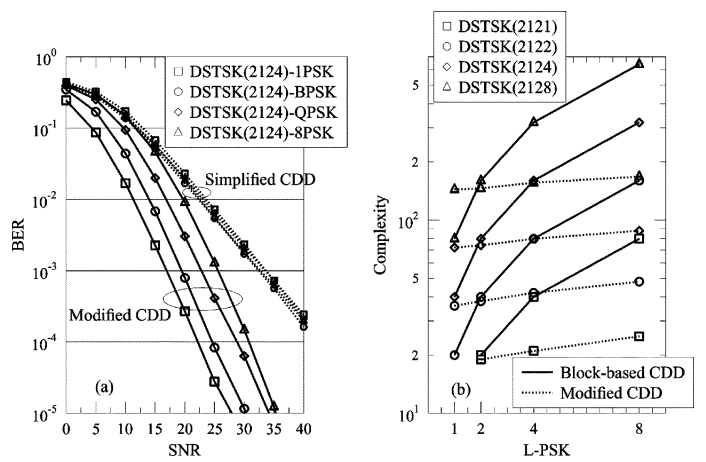

Fig. 1. BER performance and Complexity of DSTSK(212Q)-LPSK employing the proposed CDD of (9) and (10), for $f_{d}=0.001$.

the complexity curves of the proposed CDD remain near-horizontal, which suggests that the proposed CDD is capable of decoding DSTSK employing QPSK/8PSK at a similar complexity as decoding BPSK. Indeed, this is expected, because the associated complexity is only on the order of $(Q+L)$, which remains modest compared to block-based decoding that was shown to be proportional to $Q L$.

\section{Multiple-Symbol Differential Detection}

\section{A. Block-Based MSDSD}

The CDD introduced in Section III performs well under the assumption of slow fading channels, but upon increasing the Doppler frequency an irreducible error floor is formed. The multiple-symbol differential detection (MSDD) scheme detailed in [10] observes multiple received signal blocks in order to make a joint decision on the source information, so that the performance of the noncoherent receivers may be improved, especially in rapidly fading channels. Furthermore, the MSDSD was proposed in [11] in order to mitigate the complexity of MSDD.

The block-based MSDSD aided DSTM is given by [6]

$$
\sum_{i=1}^{N_{w}-1}\left\|\sum_{j=i}^{N_{w}} l_{j i} \mathbf{S}_{j}^{H} \mathbf{Y}_{j}\right\|^{2} \leq R^{2}
$$

where $l_{j i}$ represents the predictor coefficients hosted by the lower triangular matrix $\mathbf{L}$ given in [11], while $R$ denotes the MSDSD's decoding sphere radius. Let us now define the Partial Euclidean Distance (PED) component seen in (11) as

$$
d_{i}^{2}=\sum_{t=i}^{N_{w}-1}\left\|\sum_{j=t}^{N_{w}} l_{j t} \mathbf{S}_{j}^{H} \mathbf{Y}_{j}\right\|^{2}=d_{i+1}^{2}+\Delta_{i}
$$

with $i=1,2, \ldots,\left(N_{w}-1\right)$. The PED increment $\Delta_{i}$ in (12) is expressed as

$$
\Delta_{i}=\left\|l_{i i} \mathbf{X}_{i} \mathbf{Y}_{i}+\mathbf{A}_{i+1}\left(\sum_{j=i+1}^{N_{w}} l_{j i} \mathbf{A}_{j}^{H} \mathbf{Y}_{j}\right)\right\|^{2}
$$

where the accumulation matrix $\left\{\mathbf{A}_{i}\right\}_{i=1}^{N_{w}}$ is defined as

$$
\mathbf{A}_{i}=\mathbf{S}_{i} \mathbf{S}_{N_{w}}^{H}= \begin{cases}\prod_{j=i}^{N_{w}-1} \mathbf{X}_{j}^{H} & 1 \leqslant i<N_{w} \\ \mathbf{I}_{\mathbf{T}} & i=N_{w} .\end{cases}
$$

When sphere decoder visits a specific index $i$ for the first time, the optimum codeword $\mathbf{X}_{i}^{\text {opt }}$ corresponding to the smallest PED increment $\Delta_{i}$ of (13) is found. Furthermore, all the $Q L$ codewords $\left\{\mathbf{X}_{i}^{l}\right\}_{l=1}^{Q L}$ have to be ordered according to the increasing values of $\Delta_{i}$ [6], so that when the sphere decoder returns to the same index $i$, only the second-best suboptimum codeword has to be checked. Therefore, the complexity of ordering the $Q L$ candidate codewords on the basis of the block-based PED increment $\Delta_{i}$ of (13) is represented by $[(2 T+2) N T] \cdot Q L$.

\section{B. Modified MSDSD}

Observe in (13) that the PED increment $\Delta_{i}$ has a similar structure to that of the block-based CDD of (4), hence the "vectorization" of (5) may also be applied to $\Delta_{i}$ as

$$
\Delta_{i}=\left\|\hat{\mathbf{Y}}_{i}-\hat{\mathbf{H}}_{i} \chi \hat{\mathbf{K}}_{i}\right\|^{2}
$$

where the corresponding new notations are given by

$$
\begin{aligned}
& \hat{\mathbf{Y}}_{i}=\operatorname{rvec}\left(\mathbf{A}_{i+1}\left(\sum_{j=i+1}^{N_{w}} l_{j i} \mathbf{A}_{j}^{H} \mathbf{Y}_{j}\right)\right), \\
& \hat{\mathbf{H}}_{i}=-l_{i i} \cdot \mathbf{I}_{\mathbf{T}} \otimes \mathbf{Y}_{i}^{T}, \\
& \hat{\mathbf{K}}_{i}=[\underbrace{0 \cdots 0}_{\hat{q}-1}, \hat{x}_{i}, \underbrace{0 \cdots 0}_{Q-\hat{q}}] .
\end{aligned}
$$

Similar to (7), the correlation operation generates a detection vector, which may be expressed as:

$$
\hat{\mathbf{Z}}_{i}=\left(\hat{\mathbf{H}}_{i} \chi\right)^{H} \hat{\mathbf{Y}}_{i} .
$$

The $Q$-component vector $\hat{\mathbf{Z}}_{i}$ may be used to rank all the $Q L$ candidate codewords, so that the complexity of the MSDSD ranking is reduced from the order of $Q L$ to $(Q+L)$.

More explicitly, we propose the symbol-by-symbol based RC-MSDSD ranking as follows.

1) In order to rank the activation indices $\hat{q}$ first, the constellation points of the $L$-PSK modulation have to be considered in groups, which is represented by

$$
\begin{array}{r}
L=1, \quad \operatorname{dec}\left\{\operatorname{Re}\left(\mathbf{Z}_{i}\right)\right\}, \\
L=2, \quad \operatorname{dec}\left\{\left|\operatorname{Re}\left(\mathbf{Z}_{i}\right)\right|\right\}, \\
L=4, \quad \operatorname{dec}\left\{\left|\operatorname{Re}\left(\mathbf{Z}_{i}\right)\right|,\left|\operatorname{Im}\left(\mathbf{Z}_{i}\right)\right|\right\}, \\
L=8, \quad \operatorname{dec}\left\{\left|\operatorname{Re}\left(\mathbf{Z}_{i}\right)\right|,\left|\operatorname{Im}\left(\mathbf{Z}_{i}\right)\right|,\right. \\
\frac{\left|\operatorname{Re}\left(\mathbf{Z}_{i}\right)+\operatorname{Im}\left(\mathbf{Z}_{i}\right)\right|}{\sqrt{2}}, \\
\left.\frac{\left|\operatorname{Re}\left(\mathbf{Z}_{i}\right)-\operatorname{Im}\left(\mathbf{Z}_{i}\right)\right|}{\sqrt{2}}\right\}
\end{array}
$$

where dec denotes decreasingly ordering the elements of the vector. For example, if QPSK is employed, then the constellation points are grouped into $\{ \pm 1\}$ and $\{ \pm j\}$, which requires to order the absolute value of the real and the imaginary part of the detection vector, respectively.

2) The ordered dispersion matrix activation indices $\hat{q}$ should be labelled to indicate, which particular group of constellation points they refer to. 

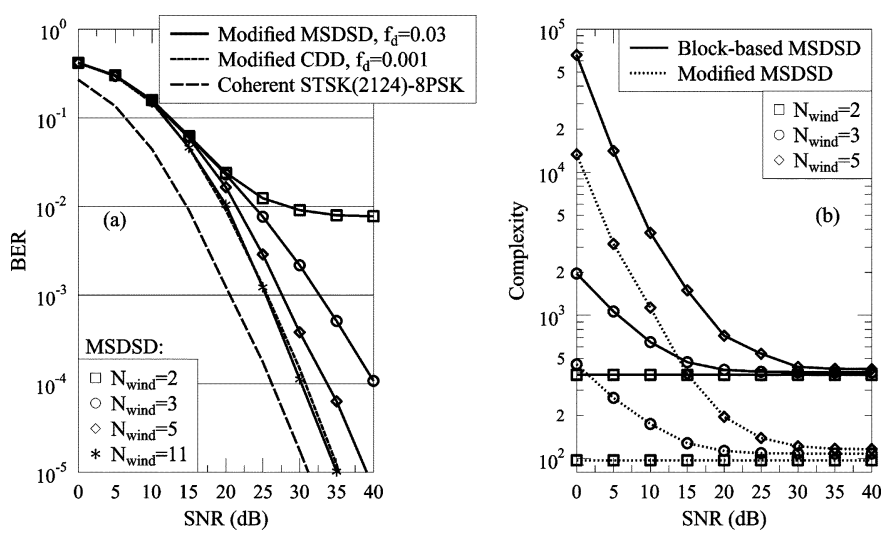

Fig. 2. BER performance and Complexity of DSTSK(2124)-8PSK employing the proposed modified MSDSD in Section IV-B, for $f_{d}=0.03$.

3) For all the $Q L$ legitimate DSTSK codewords, the first half of the candidate codewords represents the ordered dispersion matrix indices $\hat{q}$ along with their corresponding optimum constellation points. The second half is given by the indices $\hat{q}$ stored in reversed order, along with their corresponding nonoptimum solutions.

For example, assuming that QPSK signalling was employed and that the detection vector in (17) is given by $\mathbf{Z}_{i}=[2.2+3.0 j,-3.4+1.1 j]^{T}$, (18) orders the activation indices $\hat{q}$ as $[2,1,1,2]$ and labels them by $[R e, I m, R e, I m]$, because the 2 nd element in $\mathbf{Z}_{i}$ has the highest absolute real value of 3.4, while the second highest one is given by the imaginary part of the 1st element, and so on. Then the sphere decoder orders the first half of the candidate codewords as $\left\{\left(\hat{q}, \hat{x}_{i}\right)\right\}=\{(2,-1),(1, j),(1,1),(2, j)\}$, while the second half is given by $\left\{\left(\hat{q}, \hat{x}_{i}\right)\right\}=\{(2,-j),(1,-1),(1,-j),(2,1)\}$.

The complexity of the proposed MSDSD ordering contains two contributions. The first one is represented by $\left(2 T^{2}-1\right) N T Q+(2 N T-1) Q$ owing to the calculation of $\mathbf{Z}_{i}$ in (17). The second contribution is imposed by the index ordering of (18) and by the $L$-PSK demodulation, which is given by $Q,(Q+L),(2 Q+L)$ and $(4 Q+L)$ for 1-PSK, BPSK, QPSK and 8PSK, respectively.

\section{Simulation Results}

The BER performance of the proposed MSDSD is characterized in Fig. 2(a). It can be seen that the CDD aided DSTSK(2124)-8PSK scheme suffers from the usual $3 \mathrm{~dB}$ performance degradation compared to its coherent counterpart using idealized perfect channel estimation in slow fading channels. By contrast, when the normalized Doppler frequency increases to $f_{d}=0.03$, an irreducible error floor is formed. However, upon increasing the detection window length $N_{w}$, the proposed MSDSD successfully mitigates the error floor. Fig. 2(b) shows that the sphere decoder's search is typically terminated sooner at high SNRs [12]. Moreover, for the DSTSK(2124)-8PSK scheme, the proposed MSDSD exhibits a reduced complexity.

Fig. 3 portrays the complexity comparison of the block-based MSDSD of (12) and of the proposed MSDSD introduced in

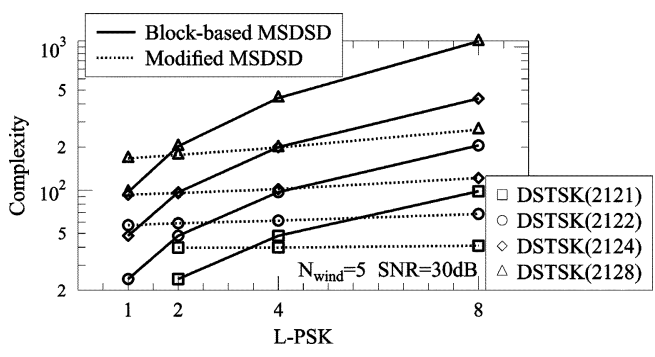

Fig. 3. Complexity comparison between the DSTSK(212Q)-LPSK employing the block-based MSDSD of (12) and employing our proposed RC-MSDSD in Section IV-B with a window length of $N_{w}=5$, for $S N R=30 \mathrm{~dB}$ and $f_{d}=0.03$.

Section IV-B for different DSTSK schemes. Similar to the CDD complexity comparison shown in Fig. 1(b), the near-horizontal complexity curves of the proposed MSDSD seen in Fig. 3 demonstrate that the proposed MSDSD is capable of decoding the DSTSK schemes employing QPSK or 8PSK at a similar complexity, to that of BPSK.

\section{CONCLUSION}

We further developed the DSTSK in [2] for avoiding the nonlinear Cayley transform. Our solution facilitates the employment of arbitrary complex-valued constellations. Our simulation results demonstrated that the proposed CDD and MSDSD are capable of achieving the optimum ML performance with a reduced complexity.

\section{REFERENCES}

[1] R. Mesleh, H. Haas, S. Sinanovic, C. W. Ahn, and S. Yun, "Spatial modulation," IEEE Trans. Veh. Technol., vol. 57, pp. 2228-2241, Jul. 2008.

[2] S. Sugiura, S. Chen, and L. Hanzo, "Coherent and differential space-time shift keying: A dispersion matrix approach," IEEE Trans. Commun., vol. 58, pp. 3219-3230, Nov. 2010.

[3] B. Hassibi and B. Hochwald, "Cayley differential unitary space-time codes," IEEE Trans. Inf. Theory, vol. 48, pp. 1485-1503, Jun. 2002.

[4] C. Yuen, Y. L. Guan, and T. T. Tjhung, "Unitary differential space-time modulation with joint modulation," IEEE Trans. Veh. Technol., vol. 56, pp. 3937-3944, Nov. 2007.

[5] J. Jeganathan, A. Ghrayeb, and L. Szczecinski, "Spatial modulation: Optimal detection and performance analysis," IEEE Commun. Lett., vol. 12, pp. 545-547, Aug. 2008.

[6] V. Pauli and L. Lampe, "On the complexity of sphere decoding for differential detection,” IEEE Trans. Inf. Theory, vol. 53, pp. 1595-1603, Apr. 2007.

[7] G. Ganesan and P. Stoica, "Differential modulation using space-time block codes," IEEE Signal Process. Lett., vol. 9, pp. 57-60, Feb. 2002.

[8] C.-S. Hwang, S. H. Nam, J. Chung, and V. Tarokh, "Differential space time block codes using nonconstant modulus constellations," IEEE Trans. Signal Process., vol. 51, pp. 2955-2964, Nov. 2003.

[9] J. Jeganathan, A. Ghrayeb, L. Szczecinski, and A. Ceron, "Space shift keying modulation for MIMO channels," IEEE Trans. Wireless Commun., vol. 8, pp. 3692-3703, Jul. 2009.

[10] P. Ho and D. Fung, "Error performance of multiple-symbol differential detection of PSK signals transmitted over correlated Rayleigh fading channels," IEEE Trans. Commun., vol. 40, pp. 1566-1569, Oct. 1992.

[11] L. Lampe, R. Schober, V. Pauli, and C. Windpassinger, "Multiplesymbol differential sphere decoding," IEEE Trans. Commun., vol. 53, pp. 1981-1985, Dec. 2005.

[12] D. Pham, K. Pattipati, P. Willett, and J. Luo, "An improved complex sphere decoder for V-BLAST systems," IEEE Signal Process. Lett., vol. 11, pp. 748-751, Sep. 2004. 